\title{
Synthesis of (-)-Canataxpropellane
}<smiles>COc1cccc2c1COC2=O</smiles><smiles>CC(C)[C@@]1(c2ccccc2)O[Si](Cl)(C(C)C)OC(c2ccccc2)(c2ccccc2)[C@H]2OC(C)(C)O[C@H]21</smiles>

B<smiles>COCC1=C(C)C(=O)C2C3(Oc4cccc(OC)c4O3)C2(c2ccccc2)C1(C)C</smiles><smiles>Cc1cccc(O)c1CO</smiles>

c

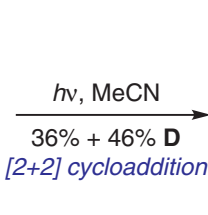

D

$R=(-)-T A D D O L]$

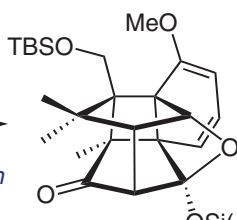

ŌSi $(i-\operatorname{Pr})(-) T A D D O L$

E 6 steps $\downarrow 24 \%$

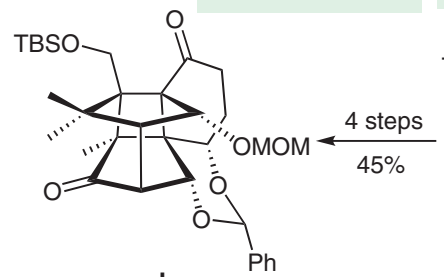

$$
\begin{aligned}
& \begin{array}{l|l}
59 \% & \text { 1. KHMDS, THF-PhMe (7:1) }
\end{array} \\
& \text { Stille } \quad-78{ }^{\circ} \mathrm{C} \text {, then } \mathrm{J},-78^{\circ} \mathrm{C} \\
& \text { carbonylative 2. } \mathrm{Et}_{3} \mathrm{~N}, \mathrm{Pd}\left(\mathrm{PPh}_{3}\right)_{4}(10 \mathrm{~mol} \%) \\
& \text { cross- } \quad \mathrm{CO} \text { (1 atm), DMF-MeOH (2:1) } \\
& \text { coupling } \\
& \text { TBSO }
\end{aligned}
$$
$\mathrm{MeO}_{2} \mathrm{C}$
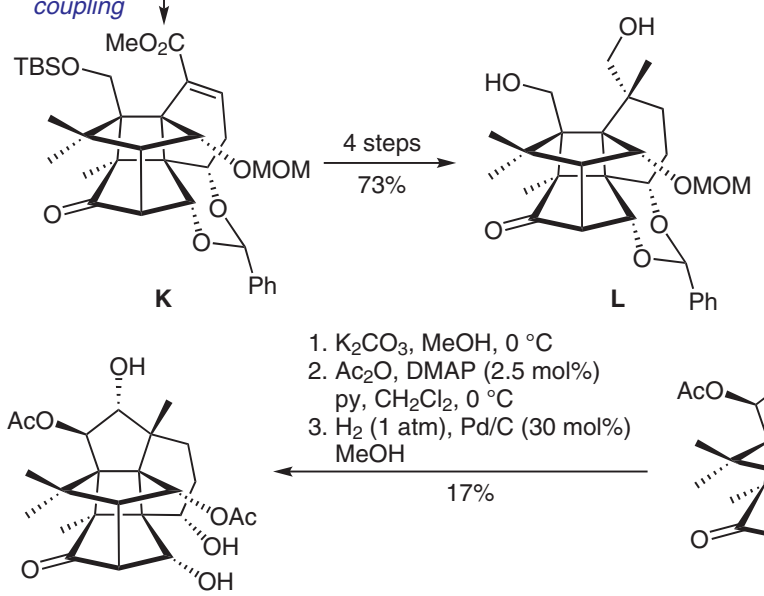

(-)-Canataxpropellane

1. $\mathrm{K}_{2} \mathrm{CO}_{3}, \mathrm{MeOH}, 0^{\circ} \mathrm{C}$ 2. $\mathrm{Ac}_{2} \mathrm{O}$, DMAP (2.5 mol\%) py, $\mathrm{CH}_{2} \mathrm{Cl}_{2}, 0^{\circ} \mathrm{C}$

3. $\mathrm{H}_{2}(1 \mathrm{~atm}), \mathrm{Pd} / \mathrm{C}(30 \mathrm{~mol} \%)$ $\mathrm{MeOH}$

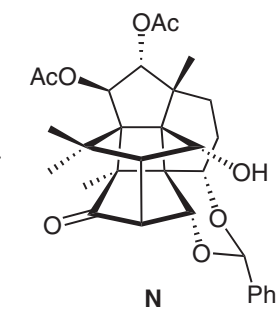

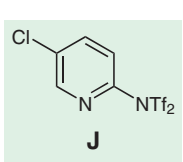

1. $(\mathrm{COCl})_{2}$, DMSO $\mathrm{CH}_{2} \mathrm{Cl}_{2},-78{ }^{\circ} \mathrm{C}$ then $\mathrm{Et}_{3} \mathrm{~N},-78$ to $0{ }^{\circ} \mathrm{C}$ 2. $\mathrm{TiCl}_{4}, \mathrm{Zn}, \mathrm{THF}-\mathrm{CH}_{2} \mathrm{Cl}_{2}$ $(27: 1)$, then py, then SM $50 \%$
Swern oxidation
pinacol coupling
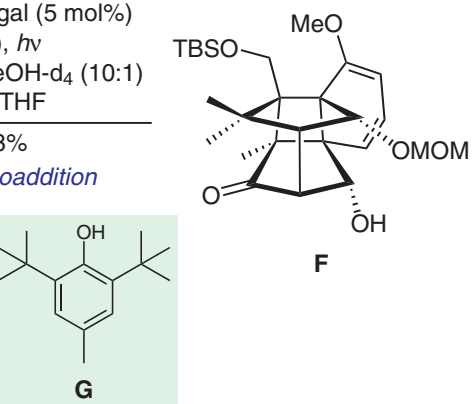

$\mathrm{OH}$
Key words

\section{(-)-canatax-} propellane

taxane diterpenoid

[2+2] cycloaddition pinacol coupling

${ }^{1} \mathrm{O}_{2}$ cycloaddition
Significance: (-)-Canataxpropellane is a taxane diterpenoid that was isolated from Taxus canadensis. Gaich and co-workers report the total synthesis of this structurally complex natural product. Key to the synthesis is an intramolecular [2+2] cycloaddition that forms the fully substituted cyclobutane.
Comment: Cyclobutane E was assembled in two steps from $\mathbf{A}$ and $\mathbf{B}$ through Diels-Alder cycloaddition and intramolecular $[2+2]$ cycloaddition. ${ }^{1} \mathrm{O}_{2}$ cycloaddition of $\mathbf{F}$ followed by reductive $\mathrm{O}-\mathrm{O}$ bond cleavage gave $\mathbf{H}$. Oxidation of diol $\mathbf{L}$ and pinacol coupling completed the carbon skeleton. 